\title{
Article \\ Potential Energy Surfaces for Noble Gas (Ar, Kr, Xe, Rn)-Propylene Oxide Systems: Analytical Formulation and Binding
}

\author{
Federico Palazzetti ${ }^{1, *(\mathbb{D}}$, Cecilia Coletti ${ }^{2} \mathbb{D}$, Alessandro Marrone ${ }^{2}$ and Fernando Pirani ${ }^{1,3} 3_{\mathbb{D}}$ \\ 1 Dipartimento di Chimica, Biologia e Biotecnologie-Università degli Studi di Perugia, 06123 Perugia, Italy; \\ fernando.pirani@unipg.it \\ 2 Dipartimento di Farmacia, Università degli Studi "G. D'Annunzio" Chieti-Pescara, 66100 Chieti, Italy; \\ ccoletti@unich.it (C.C.); alessandro.marrone@unich.it (A.M.) \\ 3 Dipartimento di Ingegneria Civile ed Ambientale, Università degli Studi di Perugia, 06215 Perugia, Italy \\ * Correspondence: federicopalazzetti@yahoo.it
}

Citation: Palazzetti, F.; Coletti, C.;

Marrone, A.; Pirani, F. Potential

Energy Surfaces for Noble Gas (Ar,

$\mathrm{Kr}, \mathrm{Xe}, \mathrm{Rn}$ )-Propylene Oxide

Systems: Analytical Formulation and

Binding. Symmetry 2022, 14, 249.

https://doi.org/10.3390/

sym14020249

Academic Editor: Haruo Hosoya

Received: 24 December 2021

Accepted: 24 January 2022

Published: 27 January 2022

Publisher's Note: MDPI stays neutral with regard to jurisdictional claims in published maps and institutional affiliations.

Copyright: (C) 2022 by the authors. Licensee MDPI, Basel, Switzerland. This article is an open access article distributed under the terms and conditions of the Creative Commons Attribution (CC BY) license (https:// creativecommons.org/licenses/by/ $4.0 /)$.

\begin{abstract}
Multidimensional potential energy surfaces for heavy noble gas-propylene oxide systems are obtained by applying the phenomenological method successfully used to describe homologous systems involving He and Ne atoms. Such potential energy surfaces, where the interaction exclusively arises from the anisotropic van der Waals interaction components, are given in an analytical form. Therefore, they can be easily used as force fields to carry out molecular simulations to evaluate spectroscopic features and the dynamical selectivity of weakly bound complexes formed by propylene oxide (a prototype chiral species) with a noble gas atom (a prototype isotropic partner) by two-body collisions under a variety of conditions. Several potential energy minima are identified on the surfaces, which are confirmed and characterized by high level ab initio calculations. The next step to further generalize this methodology is its extension to systems involving propylene oxide-diatomic molecules (as $\mathrm{H}_{2}, \mathrm{O}_{2}$ and $\mathrm{N}_{2}$ ), as well as to propylene oxide dimers.
\end{abstract}

Keywords: intermolecular forces; chirality; astrochemistry

\section{Introduction}

Van der Waals complexes, whose intermolecular bond is determined by the critical balance of size repulsion with dispersion/induction attraction, are formed in many gaseous environments by collisions between weakly interacting species, often involving atomic and/or molecular partners of different complexity. Their characterization is crucial, since it permits the evaluation of static and dynamical properties of matter under a variety of conditions [1]. Moreover, the features of weakly interacting systems are basic to define the energy and structure of precursor states, controlling the passage to, and often being coincident with, the transition state of several fundamental elementary processes. Therefore, the proper representation of multidimensional potential energy surfaces (PESs), controlled by non-covalent anisotropic interactions, where the van der Waals component plays a dominant role, turns out to be a problem of general interest. Indeed, it should be emphasized that the construction of ab initio-based PESs, involving non-covalently interacting systems, must ensure an accurate description of the long-range regions. Such an issue is crucial for the correct evolution of molecular dynamics [1-3]. The construction must rely on the use of a high-level theory and a very large number of ab initio points together with suitable interpolation procedures. The required computational cost is expected to strongly increase, becoming often prohibitive even in atom-molecule systems, especially when considering heavier atoms and complex polyatomic molecules.

Propylene oxide $\left(\mathrm{C}_{3} \mathrm{H}_{6} \mathrm{O}\right)$ is one of the simplest chiral molecules [4-10]. Its structure, involving ten atoms, has been investigated by microwave spectroscopy $[9,10]$, characterized 
by vibrational circular dichroism [11], Raman optical activity spectroscopy [12], and by $\mathrm{ab}$ initio calculations [13]. The properties of propylene oxide have been investigated by combining molecular beams and electrostatic hexapole techniques, where molecules were rotationally state-selected and aligned $[14,15]$. Synchrotron radiation was also employed to characterize the double ionization threshold [16]. The angular distribution of ionic fragments, produced by various dissociation channels of gas phase double ionized $\mathrm{C}_{3} \mathrm{H}_{6} \mathrm{O}$ molecules, has also been measured [17]. Structural isomers of propylene oxide have been investigated by quantum mechanical calculations [18,19].

The recent discovery of propylene oxide in the interstellar medium [4] increased the interest towards the basic role of such a molecule in the dynamics of elementary processes of interest for astrochemistry, and stimulated the debate on the long-standing unsolved issue about the origin of chiral selectivity in nature [20,21]. This discovery encouraged investigations on the basic features of the interaction of propylene oxide with the most abundant species in the interstellar medium, such as $\mathrm{He}$, and also $\mathrm{Ne}$ and $\mathrm{Ar}$, that are less stable than $\mathrm{He}$ [20]. Indeed, the interaction of noble gas atoms with neutral and ionic species relevant in astrochemical and interstellar media is a timely and currently much investigated subject [22].

Only limited information on the intermolecular interactions concerning propylene oxide and other closed shell partners is currently available; microwave rotational spectroscopy has been employed to characterize the structure and binding energy of the complexes with $\mathrm{Ne}$ [23], Ar [24], and $\mathrm{Kr}$ [25], and of the homochiral and heterochiral forms of propylene oxide dimers [26]. Recently, Faure et al. have constructed an accurate potential energy surface (PES) for the He-propylene oxide system [27], on which they calculated the first scattering cross sections for the rotational excitation. However, this procedure requires a high computational load, due to the number of coupled channels and of the basis functions needed to reach convergence.

Very recently, the molecular beam technique has been used to measure total (elastic and inelastic) integral cross sections, for the scattering of $\mathrm{He}$ and Ne projectiles by propylene oxide target molecules, as a function of the collision velocity [28]. A semiempirical PES, expressed as a combination of Improved Lennard-Jones (ILJ) functions, was obtained for the He-propylene oxide system defined in terms of parameters related to the polarizability components of the interacting partners (He interacting with the seven atomic centers of propylene oxide: two $\mathrm{C}$ atoms, three $\mathrm{H}$ atoms, an $\mathrm{O}$ atom, and the $\mathrm{CH}_{3}$ pseudo atom). The parameters were fine-tuned to reproduce the measured total cross section with its velocity dependence. This PES showed an excellent agreement with complete basis set quality ab initio calculations [28]. The reliability of the PES for the $\mathrm{Ne}$-propylene oxide system, obtained by simply scaling the He-propylene oxide parameters for the different polarizability of $\mathrm{Ne}$, has been confirmed by the proper description of measured cross sections. Moreover, the minima well depth, also tested on additional ab initio calculations, is consistent with the dissociation energy obtained from the analysis of experimental microwave spectra [23].

The strength points of the proposed PESs are (i) the analytical form that is crucial to perform computationally fast and reliable simulations of collision processes; and (ii) their formulation in terms of parameters related to fundamental physical properties of the interacting partners that ensures the reliability of the interaction potential in the whole configuration space. In this work, we derive, according to the framework described above, the PESs for the interaction of propylene oxide with $\mathrm{Ar}, \mathrm{Kr}, \mathrm{Xe}$, and $\mathrm{Rn}$. The involved ILJ functions are obtained by simply scaling the parameters for the increase in polarizability along the noble gas group. All the minima found for each system have been compared against new high level ab initio calculations. As observed for the $\mathrm{He}$ and Ne case [28], the adopted methodology allows the formulation of PESs which are reliable in the whole space of the relative configurations in a computationally fast way. In addition, their analytical formulation offers interesting perspectives for a profitable description of scattering and other molecular dynamics properties [29]. Therefore, this 
work represents a further step toward the characterization of the interaction potential between propylene oxide and neutral molecules of interest in astrochemistry, such as $\mathrm{H}_{2}$, $\mathrm{O}_{2}, \mathrm{~N}_{2}$, and $\mathrm{H}_{2} \mathrm{O}$, up to propylene oxide-propylene oxide interactions. The latter system is of crucial importance because it would enable the observation of possible different dynamical effects arising in homochiral and heterochiral dimers. As remarked in a recent work of classical molecular simulations on the propylene oxide-water/ice [30] system, force field investigations play a crucial role in the modelling of astrochemical environments, being experimental investigations in the corresponding extreme conditions not straightforward. Moreover, a physically meaningful formulation can provide a much deeper insight at the atomistic and molecular level into the structural and dynamical properties of the investigated systems.

\section{Potential Energy Surfaces}

In this section, we summarize the general guidelines used to obtain the PESs for the complete family of $\mathrm{Ng}-\mathrm{C}_{3} \mathrm{H}_{6} \mathrm{O}$ systems, in an internally consistent way and in analytical form, and describe their testing against ab initio calculations. As in the previous study on $\mathrm{He}$ and $\mathrm{Ne}$-propylene oxide systems [28], the total interaction potential $V$ has been represented as a sum of pair-contributions expressed through Improved Lennard Jones (ILJ) functions. All details on ILJ are given in [28] and in references therein. Each pair involves the noble gas atom $(\mathrm{Ng}=\mathrm{Ar}, \mathrm{Kr}, \mathrm{Xe}$, ad $\mathrm{Rn})$ and one of the seven centers distributed in the propylene oxide, $\mathrm{C}_{3} \mathrm{H}_{6} \mathrm{O}$, molecular frame, which are the asymmetric carbon $\mathrm{C}$, the $\mathrm{C}$ atom of the $\mathrm{CH}_{2}$ group, the three hydrogen $\mathrm{H}$ atoms of the $\mathrm{CH}$ and $\mathrm{CH}_{2}$ groups, the oxygen atom $\mathrm{O}$, and the methyl group $\mathrm{CH}_{3}$. The latter has been considered as a structure-less partner, i.e., as a sphere having the mass of the methyl group, centered on the carbon atom.

\subsection{The Improved Lennard-Jones Potential}

According to the formulation recently given in detail [28], for each neutral-neutral pair, interacting by pure van der Waals components, the ILJ function $V(r)$ has been formulated as:

$$
V(r)=\varepsilon\left(\frac{6}{n(r)-6}\left(\frac{r_{m}}{r}\right)^{n(r)}-\frac{n(r)}{n(r)-6}\left(\frac{r_{m}}{r}\right)^{6}\right),
$$

where $r$ is the $\mathrm{Ng}$-atom (or effective atom) distance, and $\varepsilon$ and $r_{m}$ are, respectively, the potential well depth and the equilibrium distance associated to the considered pair. The $n(r)$ term is given by:

$$
n(r)=\beta+4\left(\frac{r}{r_{m}}\right)^{2}
$$

where $r / r_{m}$ is the reduced distance and $\beta$ is a parameter related to the hardness of the two interacting species. In the present case, the $\beta$ value is comprised between 7.0 and 8.0 [31].

The values of the interaction parameters of all interacting partners have been estimated by a simple scaling procedure starting from those recently provided for the He-and $\mathrm{Ne}$ $\mathrm{C}_{3} \mathrm{H}_{6} \mathrm{O}$ systems according to the polarizability change along the $\mathrm{Ng}$ series [31-34]. The polarizability values of He and Ne are 0.20 and $0.40 \AA^{3}$, while those of $\mathrm{Ar}, \mathrm{Kr}$, Xe and Rn amount to $1.64,2.49,4.04$ and $5.19 \AA^{3}$, respectively [35]. The polarizability of propylene oxide, $6.2 \AA^{3}$, has been decomposed in partial contributions, corresponding to $2.10,1.10$, 0.80 and $0.35 \AA^{3}$ for $\mathrm{CH}_{3}, \mathrm{C}, \mathrm{O}$, and $\mathrm{H}$ partners, respectively. Note that $\mathrm{C}, \mathrm{H}$ and $\mathrm{O}$ are considered effective atomic centers, since their polarizabilities are different from those of isolated gas-phase atoms. The parameter values defining the PES of $\mathrm{Ar}_{-}, \mathrm{Kr}_{-}, \mathrm{Xe}-$, and $\mathrm{Rn}$-propylene oxide are reported in Tables 1-4, respectively. The predicted energy minima on each PES have been tested against the corresponding ab initio calculations and available experimental data (see the following sections). 
Table 1. Values of the equilibrium distance, $r_{m}$, and of the well depth, $\varepsilon$, used in the Improved Lennard-Jones (ILJ) model to describe individual interaction pair contributions for the Ar-propylene oxide system. The parameter $\beta$ is in the range of values typical of van der Waals interactions [22,28].

\begin{tabular}{cccc}
\hline & $\boldsymbol{\varepsilon} / \mathbf{m e V}$ & $\boldsymbol{r}_{\boldsymbol{m}} / \mathbf{A}$ & $\boldsymbol{\beta}$ \\
\hline $\mathrm{CH}_{3}-\mathrm{Ar}$ & 9.21 & 4.02 & 7.0 \\
$\mathrm{C}-\mathrm{Ar}$ & 7.19 & 3.79 & 7.0 \\
$\mathrm{O}-\mathrm{Ar}$ & 8.51 & 3.61 & 7.0 \\
$\mathrm{H}-\mathrm{Ar}$ & 3.27 & 3.51 & 8.0 \\
\hline
\end{tabular}

Table 2. Values of the equilibrium distance, $r_{m}$, and of the well depth, $\varepsilon$, used in the ILJ model to describe individual interaction pair contributions for the Kr-propylene oxide system. The parameter $\beta$ is in the range of values typical of van der Waals interactions $[22,28]$.

\begin{tabular}{cccc}
\hline & $\varepsilon / \mathbf{m e V}$ & $\boldsymbol{r}_{\boldsymbol{m}} / \mathbf{\AA}$ & $\beta$ \\
\hline $\mathrm{CH}_{3}-\mathrm{Kr}$ & 11.78 & 4.11 & 7.0 \\
$\mathrm{C}-\mathrm{Kr}$ & 8.73 & 3.91 & 7.0 \\
$\mathrm{O}-\mathrm{Kr}$ & 9.72 & 3.76 & 7.0 \\
$\mathrm{H}-\mathrm{Kr}$ & 3.59 & 3.69 & 8.0 \\
\hline
\end{tabular}

Table 3. Values of the equilibrium distance, $r_{m}$, and of the well depth, $\varepsilon$, used in the ILJ model to describe individual interaction pair contributions for the Xe-propylene oxide system. The parameter $\beta$ is in the range of values typical of van der Waals interactions [22,28].

\begin{tabular}{cccc}
\hline & $\boldsymbol{\varepsilon} / \mathbf{m e V}$ & $\boldsymbol{r}_{\boldsymbol{m}} / \mathbf{\AA}$ & $\beta$ \\
\hline $\mathrm{CH}_{3}-\mathrm{Xe}$ & 14.25 & 4.26 & 7.0 \\
$\mathrm{C}-\mathrm{Xe}$ & 9.93 & 4.09 & 7.0 \\
$\mathrm{O}-\mathrm{Xe}$ & 10.19 & 3.97 & 7.0 \\
$\mathrm{H}-\mathrm{Xe}$ & 3.67 & 3.93 & 8.0 \\
\hline
\end{tabular}

Table 4. Values of the equilibrium distance, $r_{m}$, and of the well depth, $\varepsilon$, used in the ILJ model to describe individual interaction pair contributions for the Rn-propylene oxide system. The parameter $\beta$ is in the range of values typical of van der Waals interactions [22,28].

\begin{tabular}{cccc}
\hline & $\varepsilon / \mathbf{m e V}$ & $\boldsymbol{r}_{\boldsymbol{m}} / \mathbf{\AA}$ & $\boldsymbol{\beta}$ \\
\hline $\mathrm{CH}_{3}-\mathrm{Rn}$ & 16.80 & 4.34 & 7.0 \\
$\mathrm{C}-\mathrm{Rn}$ & 11.37 & 4.19 & 7.0 \\
$\mathrm{O}-\mathrm{Rn}$ & 11.39 & 4.10 & 7.0 \\
$\mathrm{H}-\mathrm{Rn}$ & 3.96 & 4.07 & 8.0 \\
\hline
\end{tabular}

\subsection{Ab Initio Calculations}

Minimum energy points for all the investigated systems were characterized by high level ab initio calculations performed at MP2 and CCSD(T) levels of theory by using Gaussian16 [36]. In detail, initial structures were taken as the minima obtained by the analytical ILJ potential energy surfaces. To identify the orientation of the hydrogen atoms in the methyl group, which is considered as a pseudo-atom in the ILJ model, a preliminary optimization of the hydrogen atoms position was carried out at the MP2/aug-cc-pVDZ level of theory for each starting structure. Subsequent calculations were then performed on the propylene oxide- $\mathrm{Ng}$ systems, with propylene oxide taken as a rigid moiety, at MP2/aug-cc-pVnZ ( $n=2,3,4$ ) (for Ar) or MP2/aug-cc-pVnZ-PP ( $=2,3,4$ ) (for $\mathrm{Kr}$, Xe and $\mathrm{Rn}$ ) to extrapolate the complete basis set (CBS) limit, by using a mixed exponential/Gaussian function [37]. A preliminary calculation for the propylene oxide-Kr system using the corresponding all electron aug-cc-pVnZ basis sets showed that minima obtained with the use of pseudo potentials presents negligible differences in the geometry (up to 
$0.012 \AA$ ) and in energy (up to $0.5 \mathrm{meV}$ ). All optimizations were performed on the counterpoise corrected potential energy surface [38] to account for the basis set superposition error (BSSE). CCSD $(\mathrm{T})$ corrections to the MP2 CBS energies, $\triangle C C S D(T)$, were evaluated with the aug-cc-pVTZ basis set: $\triangle C C S D(T)=E_{\operatorname{CCSD}(T)}^{a u g-c c-p V T Z}-E_{M P 2}^{a u g-c c-p V T Z}$. This term was then used to calculate $E_{C C S D(T)}^{C B S}$, the CBS complex energy at the $\operatorname{CCSD}(\mathrm{T})$ level, as $E_{C C S D(T)}^{C B S} \approx E_{M P 2}^{C B S}+\triangle C C S D(T)$. For non-covalent adducts, it was indeed noted that higher order correlation effects have very little sensitivity to the basis set size (for not too small basis sets including diffuse functions) [39].

\section{Results and Discussion}

The reference frame adopted to represent each PES is shown in Figure 1: the noble gas atom-propylene oxide molecule system is embedded in a $x y z$ Cartesian reference frame, where $x, y$, and $z$ correspond to the principal molecular axes of inertia $\mathrm{b}, \mathrm{c}$, and a, respectively. The origin of the axes is fixed on the center of mass of the molecule. The spherical coordinates $R, \theta$ and $\phi$ define the noble gas atom position with respect to propylene oxide; $R$ indicates the distance between the noble gas and the molecule center of mass, and $\theta$ and $\phi$ are the polar angles.

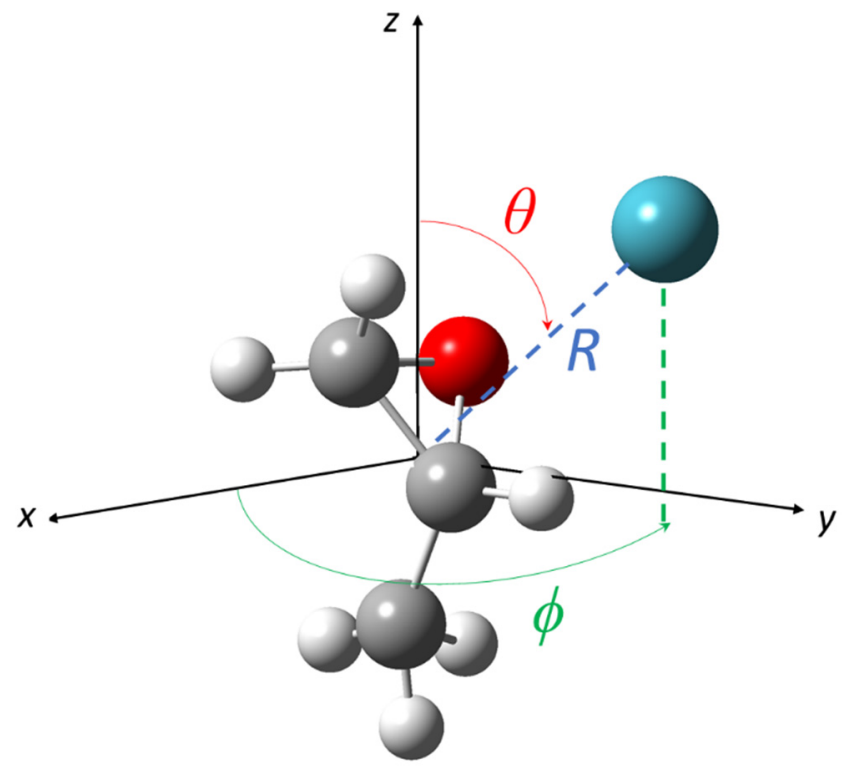

Figure 1. Coordinate frame adopted for noble gas-propylene oxide systems. The radial coordinate $R$ and the angular coordinates $\theta$ and $\phi$ are reported.

A thorough minima search on the PESs indicates that each investigated system is characterized by five minima of comparable energy, whose coordinates are reported in Tables 5-8, for the Ar-, $\mathrm{Kr}-, \mathrm{Xe}$-, and $\mathrm{Rn}$ - propylene oxide systems, respectively. Tables also report coordinates and binding energies obtained at the CCSD(T)/CBS level, as described above (energy values obtained by each considered level of theory/basis sets can be found in Tables S1-S4 in the Supporting Information).

Figure 2 reports the geometries of the five minima for $A r-\mathrm{C}_{3} \mathrm{H}_{6} \mathrm{O}$. The figure can be considered as a reference also for the other systems since the geometries of the five minima are basically very similar for the noble gases investigated here, as well as for the previously characterized $\mathrm{Ne}-\mathrm{C}_{3} \mathrm{H}_{6} \mathrm{O}$ system [28]. In particular, the equilibrium distances grow with the mass (or size) of the $\mathrm{Ng}$ atom, but the angular coordinates remain approximately the same. Furthermore, as expected, the stability of the systems is enhanced along the noble gas atoms series, according to the increase in the $\mathrm{Ng}$ atom polarizability. In detail, the global minimum configuration of Ar-propylene oxide is the same as those for heavier noble gases (see Tables 5-8 and Figures S1-S3 for Kr-, Xe-, and Rn- propylene oxide, respectively), 
and for Ne-propylene oxide [28] and corresponds to the noble gas directed toward the asymmetric carbon, from the side of the $\mathrm{CH}_{2}$ group. For the other minima, the ranking in energy might vary depending on the system. It must be noted, however, that, with the exception of the fifth minimum (see the following), they all lie very close in energy. In Minimum 2, the noble gas is directed toward the $\mathrm{O}-\mathrm{CH}_{2}$ bond in a perpendicular fashion, and the equilibrium distance is about $0.2 \AA$ shorter than Minimum 1 , while the well depth is slightly lower with respect to the global minimum. Minimum 3 and Minimum 4 present a similar well depth, significantly lower than Minimum 1. In Minimum 3, the noble gas is directed toward the $\mathrm{O}$ atom, while the orientation of the noble gas in Minimum 4 is somewhat opposite to that in Minimum 1, with the noble gas approaching the central carbon from the side of the oxygen atom. It is interesting to notice that this "hierarchy", emphasized by Ar, also holds in systems with $\mathrm{Kr}, \mathrm{Xe}$, and $\mathrm{Rn}$. However, there is an inversion in the energy ranking between Minimum 3 and 4 . In the Ar case, these two configurations are almost isoenergetic. The fifth minimum is located at a reduced distance, $r / r_{m}$ of ca. 1.08, and is significantly higher in energy with respect to the global minimum, ca. $8 \mathrm{meV}$ for $\mathrm{Ar}$, $11 \mathrm{meV}$ for $\mathrm{Kr}, 13 \mathrm{meV}$ for $\mathrm{Xe}$, and $16 \mathrm{meV}$ for $\mathrm{Rn}$ : the direction of the noble gas is almost coplanar to the propylene oxide ring, toward the oxygen atom.

Table 5. Minimum geometries and interaction energies for the Ar-propylene oxide system obtained on the present PES and by ab initio calculations (in parenthesis).

\begin{tabular}{cccccc}
\hline & Minimum 1 & Minimum 2 & Minimum 3 & Minimum 4 & Minimum 5 \\
\hline \multirow{2}{*}{$\theta$} & 98.8 & 87.6 & 49.7 & 100.4 & 43.1 \\
& $(99.8)$ & $(81.3)$ & $(50.0)$ & $(99.4)$ & $(30.5)$ \\
\hline \multirow{2}{*}{$\phi$} & 22.6 & 254.7 & 114.8 & 156.5 & 255.6 \\
& $(10.5)$ & $(259.1)$ & $(106.7)$ & $(66.1)$ & $(247.4)$ \\
\hline \multirow{2}{*}{$R(\AA)$} & 3.93 & 3.74 & 4.06 & 3.88 & 4.24 \\
& $(3.83)$ & $(3.75)$ & $(3.87)$ & $(3.67)$ & $(4.15)$ \\
\hline \multirow{2}{*}{$V(\mathrm{meV})$} & -34.6 & -34.1 & -31.8 & -31.7 & -26.9 \\
& $(-36.8)$ & $(-36.0)$ & $(-40.2)$ & $(-40.3)$ & $(-33.0)$ \\
\hline
\end{tabular}

Table 6. Minimum geometries and interaction energies for the Kr-propylene oxide system obtained on the present PES and by ab initio calculations (in parenthesis).

\begin{tabular}{cccccc}
\hline & Minimum 1 & Minimum 2 & Minimum 3 & Minimum 4 & Minimum 5 \\
\hline \multirow{2}{*}{$\theta$} & 99.9 & 88.8 & 49.9 & 101.7 & 43.2 \\
& $(99.3)$ & $(80.8)$ & $(49.9)$ & $(98.8)$ & $(31.5)$ \\
\hline \multirow{2}{*}{$\phi$} & 21.2 & 254.1 & 115.9 & 157.9 & 254.9 \\
& $(12.0)$ & $(257.7)$ & $(108.7)$ & $(157.0)$ & $(246.4)$ \\
\hline \multirow{2}{*}{$R(\AA)$} & 4.06 & 3.88 & 4.21 & 4.02 & 4.38 \\
& $(3.93)$ & $(3.81)$ & $(3.97)$ & $(3.77)$ & $(4.28)$ \\
\hline \multirow{2}{*}{$V(\mathrm{meV})$} & -42.2 & -40.9 & -37.0 & -38.2 & -31.7 \\
& $(-44.2)$ & $(-44.6)$ & $(-49.2)$ & $(-49.0)$ & $(-39.8)$ \\
\hline
\end{tabular}

Blanco et al. investigated Ar-propylene oxide and $\mathrm{Kr}$-propylene oxide by rotational spectroscopy $[24,25]$ and determined, by combining experimental and theoretical information by ab initio calculations at the MP2/6-311++G** level of theory, one adduct for each system. For Ar-propylene oxide, an experimental value of $\mathrm{R}=3.773 \AA$ and a calculated one of $R=3.711 \AA$ were obtained [23], which very well matched with the $3.74 \AA$ of Minimum 2 derived in the present formulation, together with a consistent orientation (direct comparison with the angles is not available). For $\mathrm{Kr}$-propylene oxide, an experimental value of $\mathrm{R}=3.878 \AA$ and a calculated one of $\mathrm{R}=3.905 \AA$ were obtained [25] in excellent agreement with $\mathrm{R}=3.88 \AA$ of Minimum 2, which also very well matches the experimental angles, $\theta=76^{\circ}$ (expt.) vs. $\theta=88.8^{\circ}$ (present) and $\phi=263^{\circ}$ (expt.) vs. $\phi=254.1^{\circ}$ (present). In the 
above-mentioned references, dissociation energies $D_{0}$ were also indirectly (i.e., by using an approximate formula starting from the experimental centrifugal distortion constant) obtained. Their values (31 meV for $\mathrm{Ar}-\mathrm{C}_{3} \mathrm{H}_{6} \mathrm{O}$ and $38.3 \mathrm{meV}$ for $\mathrm{Kr}-\mathrm{C}_{3} \mathrm{H}_{6} \mathrm{O}$ ) cannot be directly compared to the $D_{e}$ we provide here, because $D_{0}$ data include zero-point energy contributions. Therefore, we performed ab initio calculations at the MP2/aug-cc-pVTZ level of theory and evaluated zero-point energy corrections of 2.4 and $5.5 \mathrm{meV}$ for Ar and $\mathrm{Kr}$, respectively, which, summed to our $D_{e}$ values, lead to $D_{0}$ energies very close to those of Refs. [24,25].

Table 7. Minimum geometries and interaction energies for the Xe-propylene oxide system obtained on the present PES and by ab initio calculations (in parenthesis).

\begin{tabular}{cccccc}
\hline & Minimum 1 & Minimum 2 & Minimum 3 & Minimum 4 & Minimum 5 \\
\hline \multirow{2}{*}{$\theta$} & 101.2 & 90.1 & 50.2 & 103.1 & 41.9 \\
& $(98.7)$ & $(79.0)$ & $(49.9)$ & $(97.5)$ & $(33.0)$ \\
\hline \multirow{2}{*}{$\phi$} & 20.0 & 253.4 & 117.3 & 159.6 & 254.0 \\
& $(13.2)$ & $(256.2)$ & $(110.8)$ & $(157.0)$ & $(244.6)$ \\
\hline \multirow{2}{*}{$R(\AA)$} & 4.25 & 4.07 & 4.42 & 4.21 & 4.59 \\
& $(4.10)$ & $(3.97)$ & $(4.12)$ & $(3.94)$ & $(4.39)$ \\
\hline \multirow{2}{*}{$V(\mathrm{meV})$} & -48.1 & -46.0 & -40.2 & -43.1 & -34.9 \\
& $(-53.2)$ & $(-55.2)$ & $(-60.2)$ & $(-59.7)$ & $(-49.2)$ \\
\hline
\end{tabular}

Table 8. Minimum geometries and interaction energies for the Rn-propylene oxide system obtained on the present PES and by ab initio calculations (in parenthesis).

\begin{tabular}{cccccc}
\hline & Minimum 1 & Minimum 2 & Minimum 3 & Minimum 4 & Minimum 5 \\
\hline \multirow{2}{*}{$\theta$} & 102.8 & 90.8 & 50.5 & 103.8 & 40.8 \\
& $(98.6)$ & $(79.7)$ & $(49.8)$ & $(96.9)$ & $(33.8)$ \\
\hline \multirow{2}{*}{$\phi$} & 19.4 & 253.1 & 118.1 & 160.4 & 253.6 \\
& $(13.8$ & $(255.1)$ & $(112.0)$ & $(156.5)$ & $(243.2)$ \\
\hline \multirow{2}{*}{$R(\AA)$} & 4.36 & 4.18 & 4.54 & 4.32 & 4.72 \\
& $(4.15)$ & $(4.01)$ & $(4.17)$ & $(3.99)$ & $(4.43)$ \\
\hline \multirow{2}{*}{$V(\mathrm{meV})$} & -55.1 & -52.6 & -45.1 & -49.4 & -39.5 \\
& $(-59.9)$ & $(-63.7)$ & $(-67.6)$ & $(-67.1)$ & $(-55.2)$ \\
\hline
\end{tabular}

$\mathrm{Ab}$ initio calculations confirm the global picture given by the analytical PESs; for each system, there are five minima, four of which are very close in energy, and a slightly less bound adduct. Ab initio binding energies are higher than those predicted by the analytical PES and the discrepancy gets larger with the $\mathrm{Ng}$ mass, but in most cases the difference is within the accuracy of both methods (the accuracy of ab initio values at this level of theory is within $20 \mathrm{meV}$ (less than $0.5 \mathrm{kcal} / \mathrm{mol}$ ). The agreement in the minima geometries is also remarkably good. Apart from some exceptions, discrepancies lie within $0.15 \AA$ for distances and 15 degrees for angles. With the minima being very close in energy, it is not surprising that the energy ordering might be different in the two approaches. However, a regular pattern is also followed in this case: ab initio Minima 3 and 4 are predicted to be more stable than Minima 1 and 2, the lowest ones on the present PESs.

In order to emphasize strength and anisotropy of the interaction and important topography details of obtained PESs, we show contour maps of the intermolecular potential at the equilibrium distance of the absolute minimum and with a distance increase of $10 \%$, respectively, in Figures 3 and 4 for $\mathrm{Ar}-\mathrm{C}_{3} \mathrm{H}_{6} \mathrm{O}$, in Figures 5 and 6 for $\mathrm{Kr}-\mathrm{C}_{3} \mathrm{H}_{6} \mathrm{O}$, in Figures 7 and 8 for $\mathrm{Xe}-\mathrm{C}_{3} \mathrm{H}_{6} \mathrm{O}$, and in Figures 9 and 10 for the $\mathrm{Rn}-\mathrm{C}_{3} \mathrm{H}_{6} \mathrm{O}$ system. For each system, at the equilibrium distance of the absolute minimum, the minimum energy path (MEP) is also depicted. Contour maps obtained at shorter distances are also reported in the SI for Arand $\mathrm{Kr}$-propylene oxide (Figures S4 and S5). 


\section{5}

\section{3}

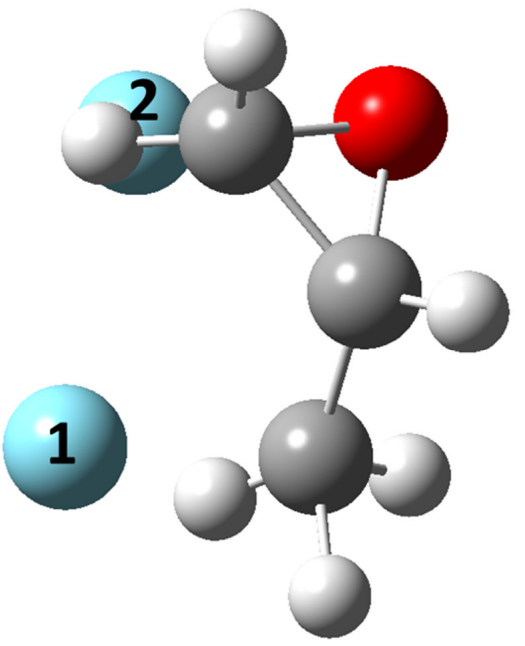

4

Figure 2. Minimum geometries for the Ar-propylene oxide system on the present PES. The numbering of the Ar atom corresponds to the minima reported in Table 5.

Propylene oxide - Ar $3.9 \AA$
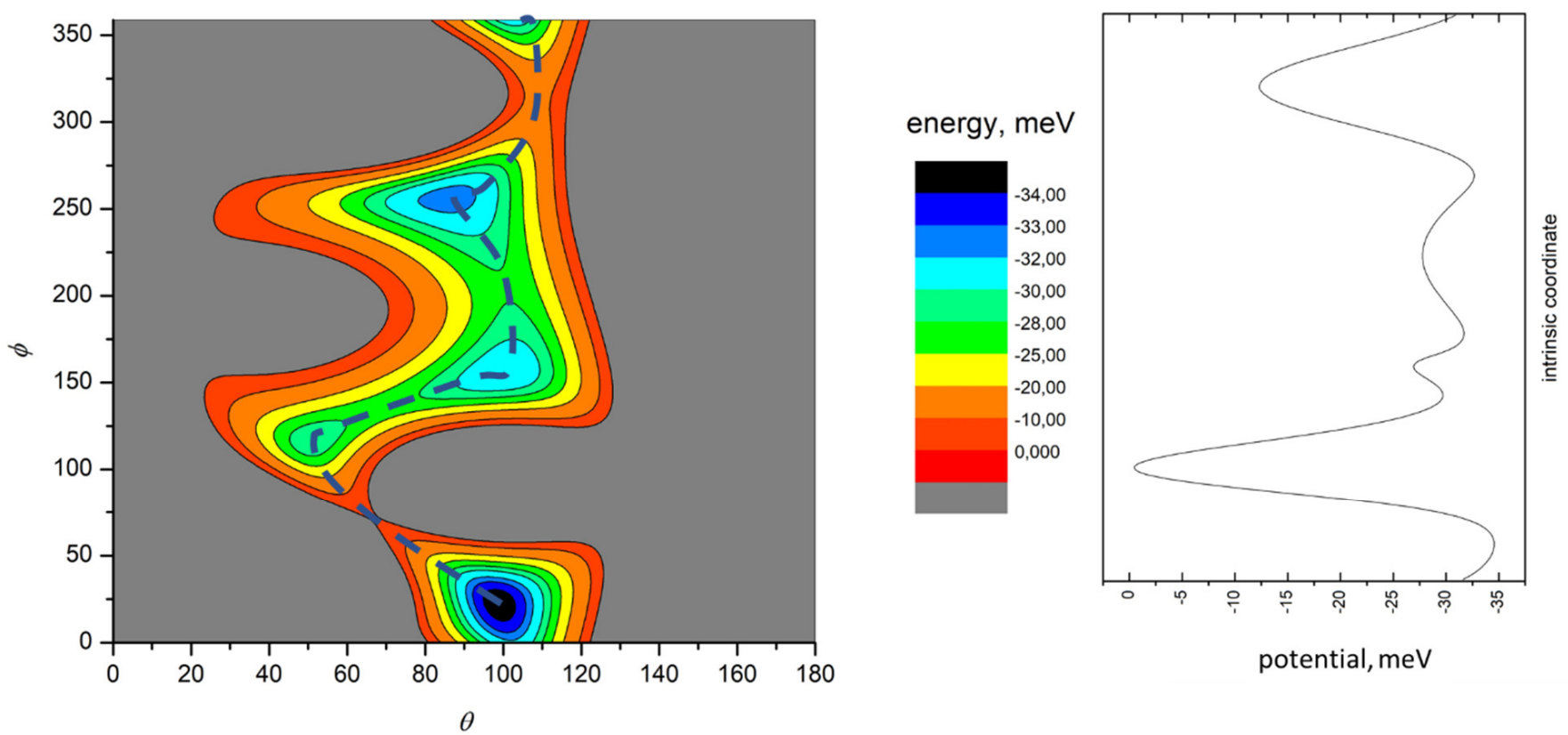

Figure 3. Color contour map for the Ar-propylene oxide system at $R=3.9 \AA$ (left panel). The axes report the angles $\theta$ and $\phi$ in degrees, while a color scale reports the potential energy in meV. The blue dashed line indicates the minimum energy path. The right panel shows the energy profile of the minimum energy path. 


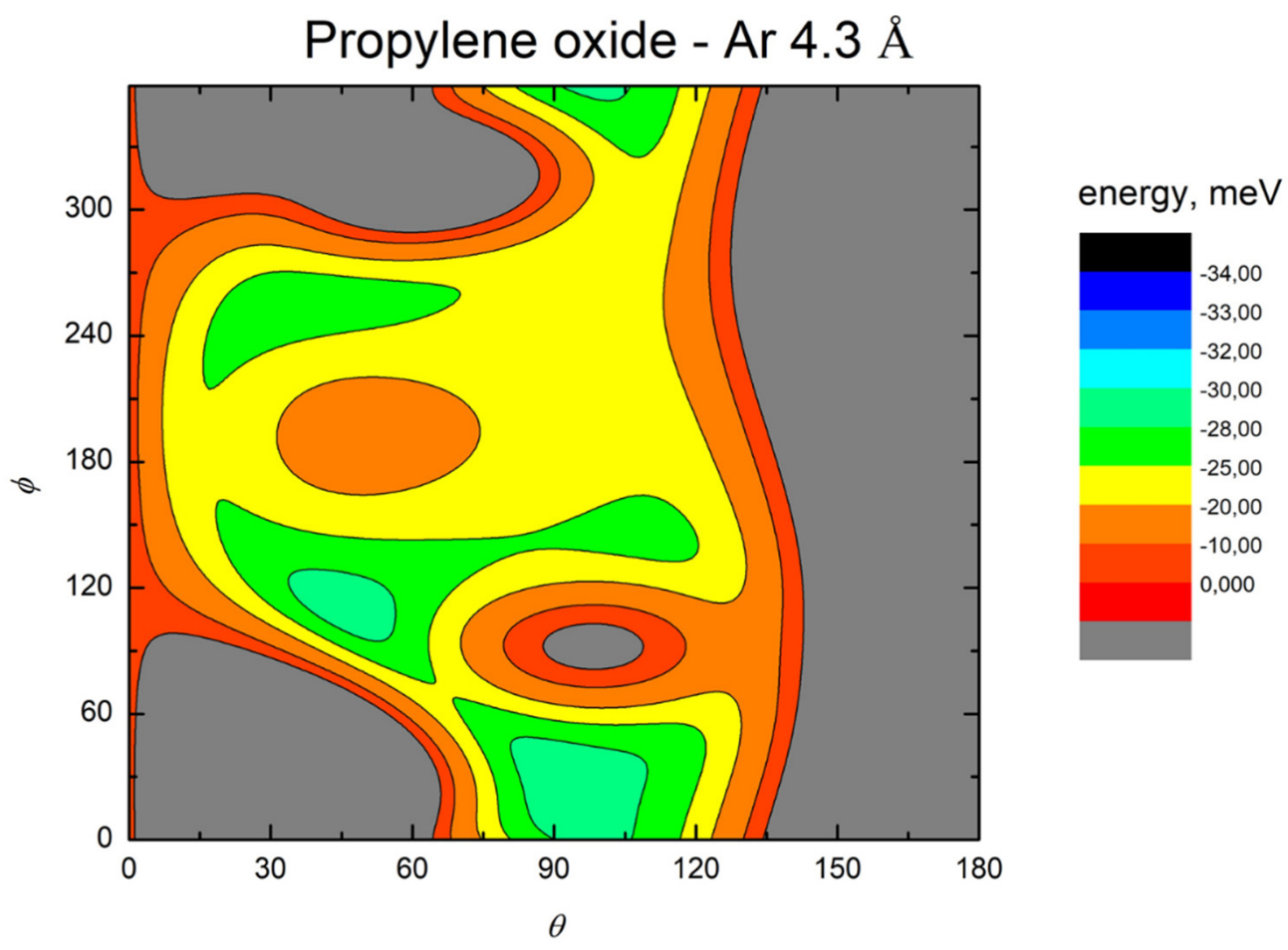

Figure 4. Color contour map for the Ar-propylene oxide system at $R=4.3 \AA$. The axes report the angles $\theta$ and $\phi$ in degrees, while a color scale indicates the potential energy in meV.
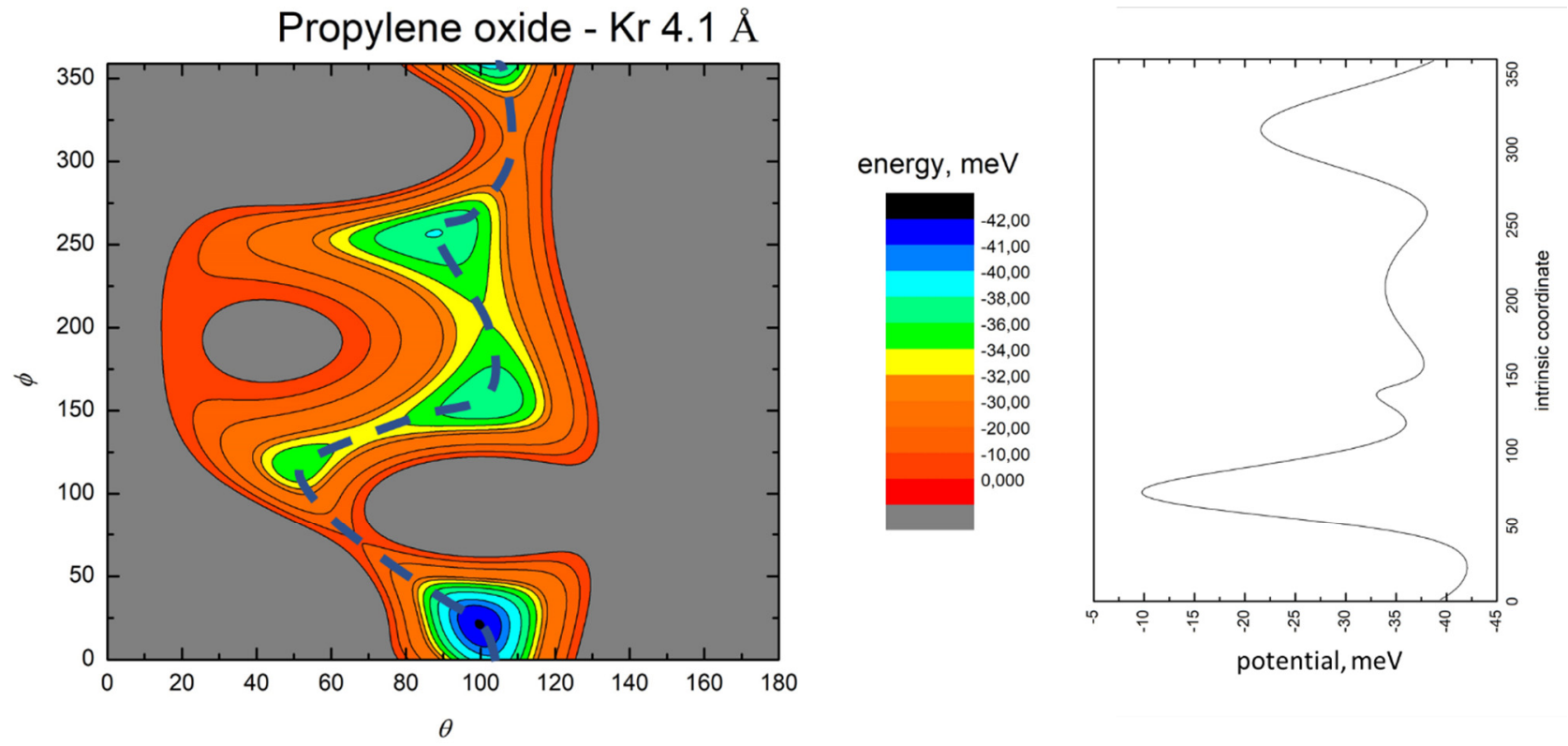

Figure 5. Color contour map for the $\mathrm{Kr}$-propylene oxide system at $R=4.1 \AA$ (left panel). The axes report the angles $\theta$ and $\phi$ in degrees, while a color scale indicates the potential energy in meV. The blue dashed line indicates the minimum energy path. The right-hand panel shows the energy profile of the minimum energy path. 


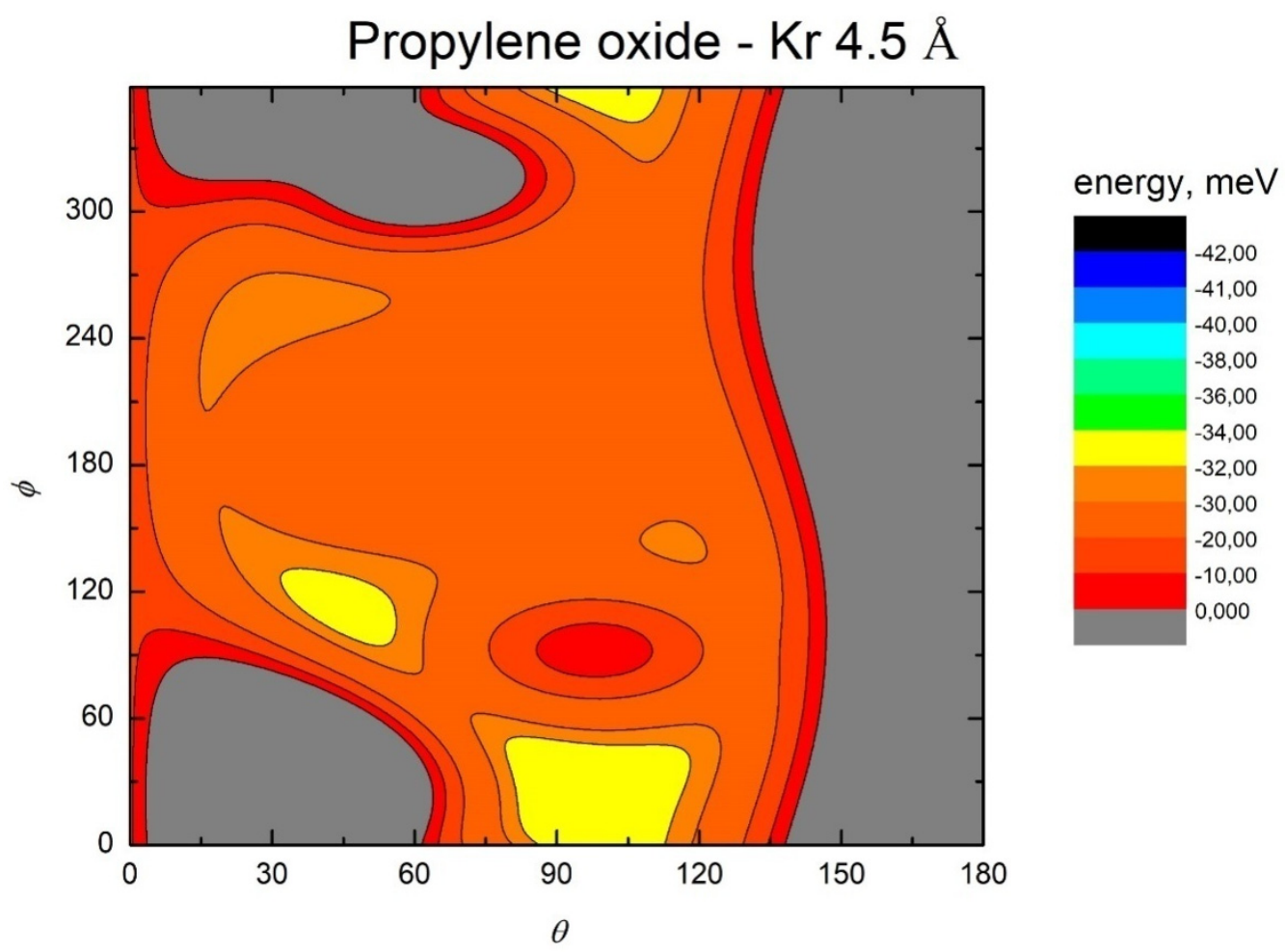

Figure 6. Color contour map for the Kr-propylene oxide system at $R=4.5 \AA$. The axes report the angles $\theta$ and $\phi$ in degrees, while a color scale indicates the potential energy in meV.

\section{Propylene oxide - Xe $4.3 \AA$}
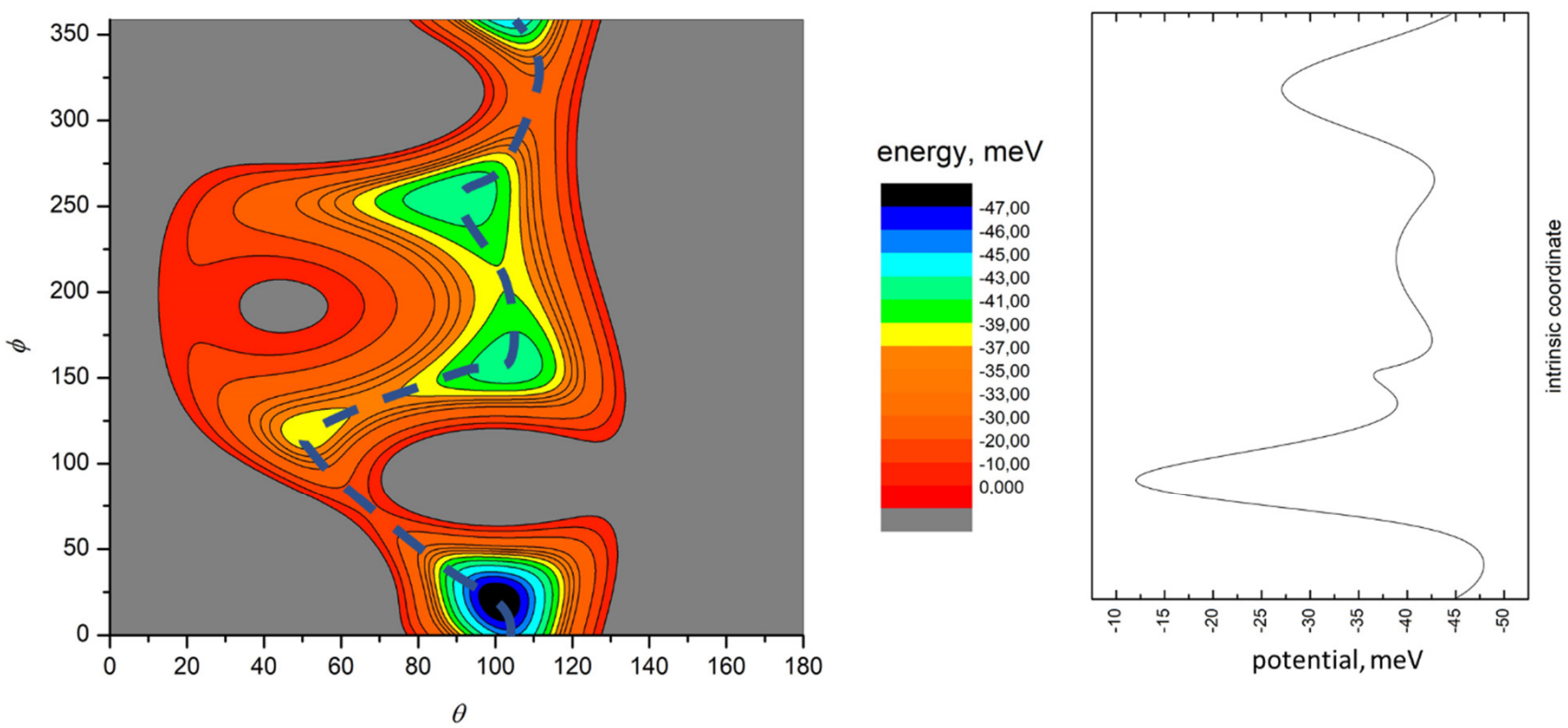

Figure 7. Color contour map for the Xe-propylene oxide system at $R=4.3 \AA$ (left panel). The axes report the angles $\theta$ and $\phi$ in degrees, while a color scale indicates the potential energy in meV. The blue dashed line indicates the minimum energy path. The right-hand panel shows the energy profile of the minimum energy path. 


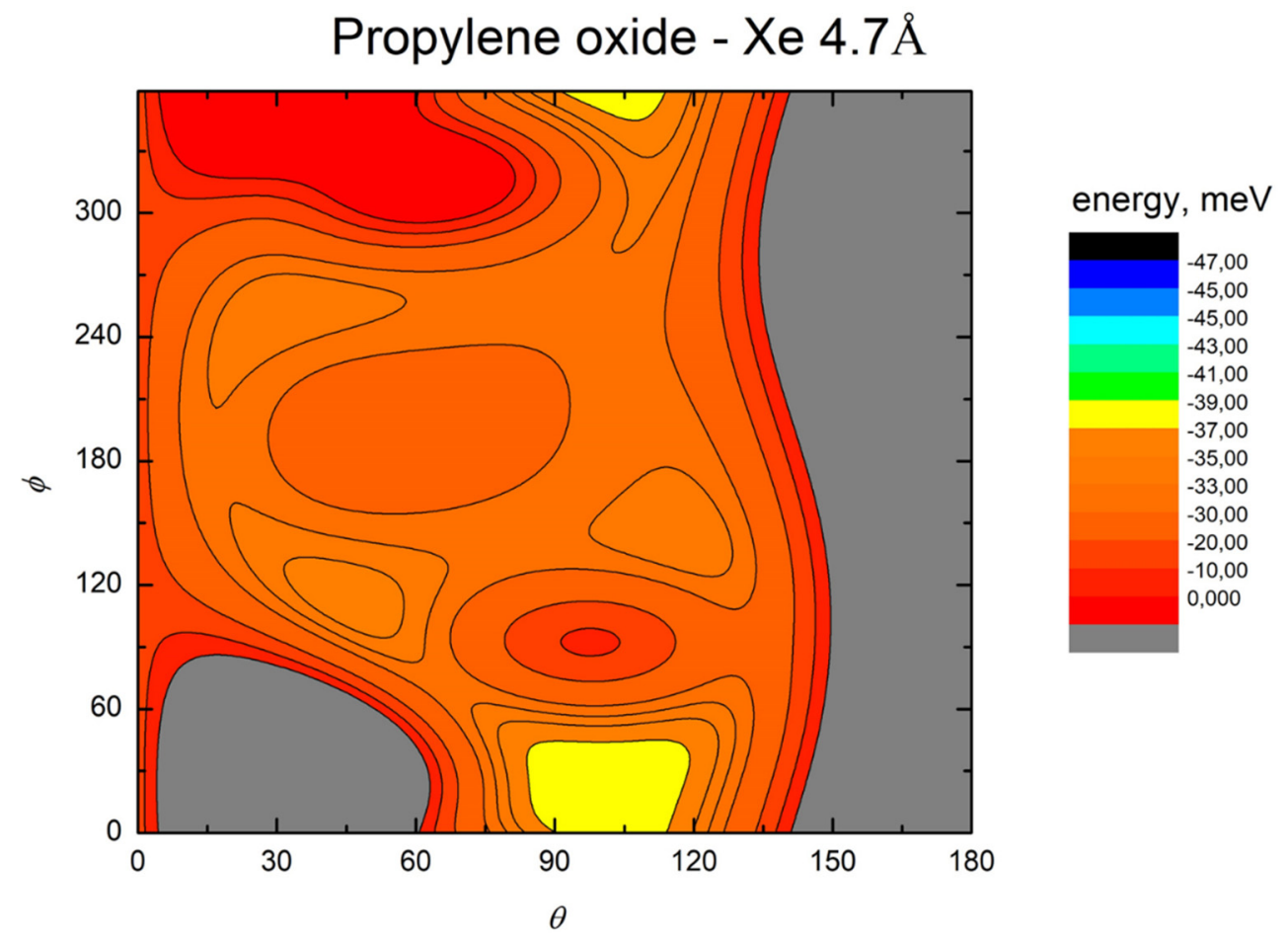

Figure 8. Color contour map for the Kr-propylene oxide system at $R=4.7 \AA$ (left panel). The axes report the angles $\theta$ and $\phi$ in degrees, while a color scale indicates the potential energy in $\mathrm{meV}$.

\section{Propylene oxide - Rn $4.4 \AA$}
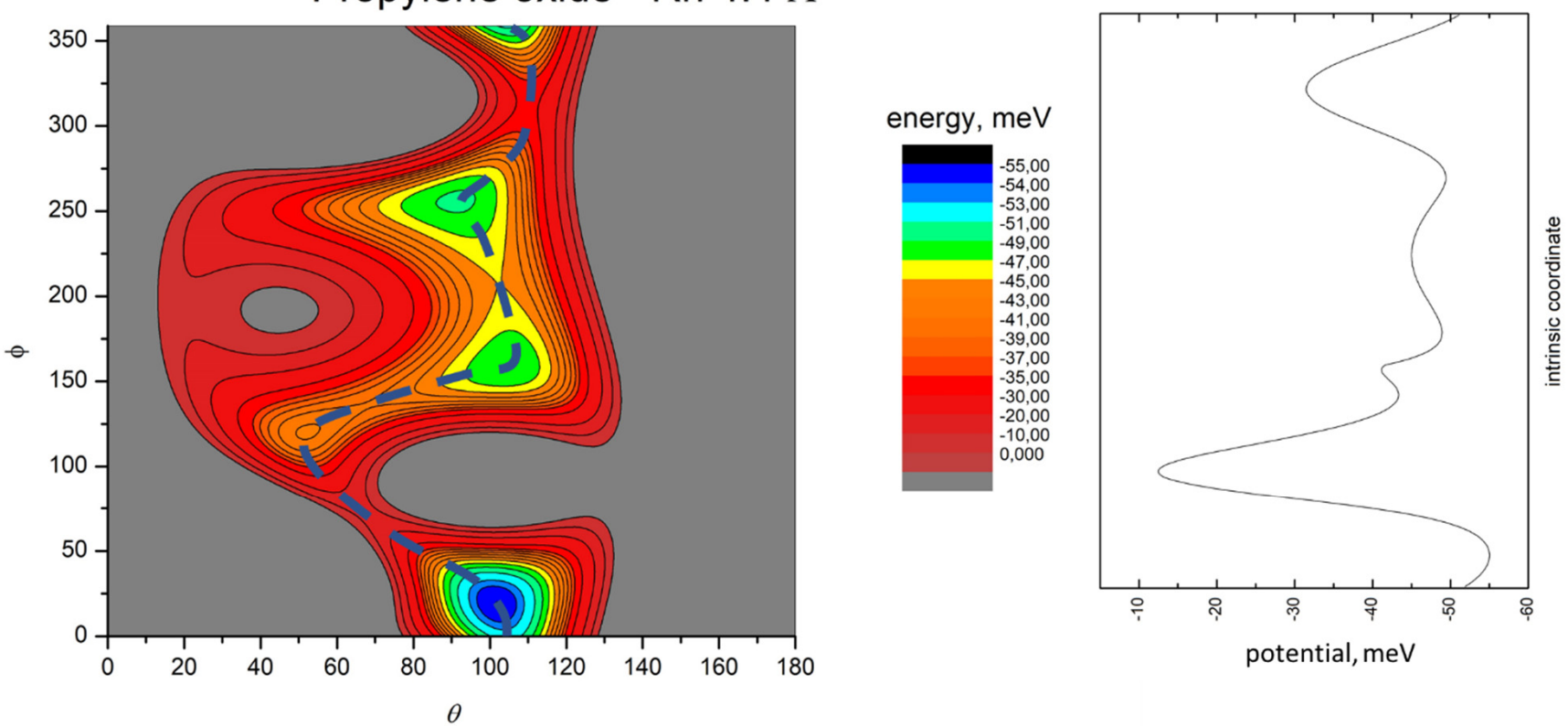

Figure 9. Color contour map for the Rn-propylene oxide system at $R=4.4 \AA$ (left panel). The axes report the angles $\theta$ and $\phi$ in degrees, while a color scale indicates the potential energy in meV. The blue dashed line indicates the minimum energy path. The right-hand panel shows the energy profile of the minimum energy path. 


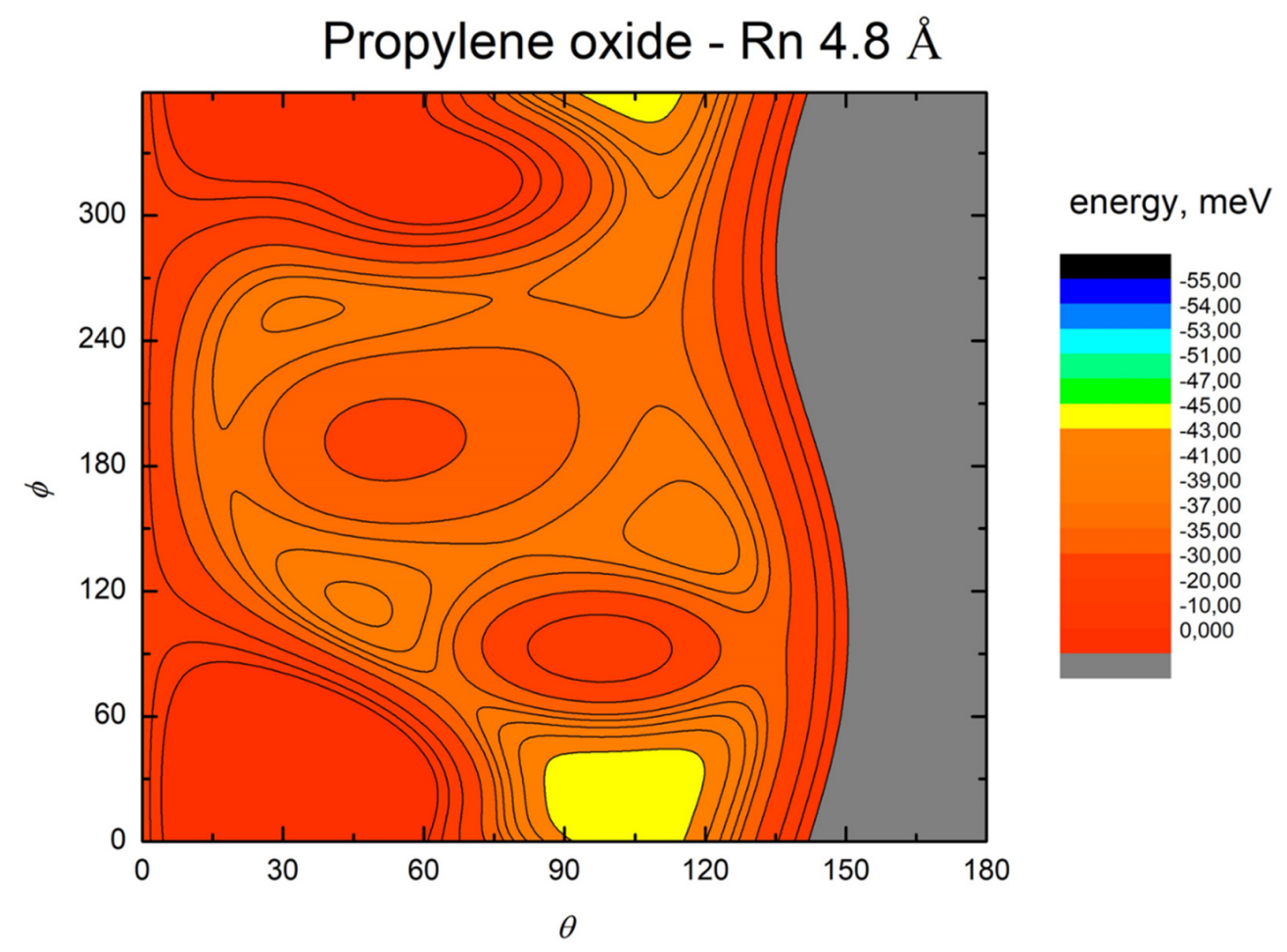

Figure 10. Color contour map for the $\mathrm{Rn}$-propylene oxide system at $R=4.8 \AA$. The axes report the angles $\theta$ and $\phi$ in degrees, while a color scale indicates the potential energy in meV.

The MEP is particularly useful to highlight the energy barriers separating the minima on the PES and thus controlling the possibility of geometry changes of the interacting system. This is an important feature, since the minima are very close in energy, and they arise at different separation distances and geometries. Therefore, if the barriers are sufficiently high, the probability of populating the minima does not solely depend on their relative magnitude, but also on the long-range orientation effects. The figures show that, as previously evidenced in the $\mathrm{He}$ - and $\mathrm{Ne}$ - propylene oxide case, the barrier heights grow larger with the $\mathrm{Ng}$ mass. This means that, particularly for the heavier systems, the approaching collision partners can get trapped at long range in specific minima configurations whose barriers cannot be overcome even at room temperature. Note that this occurs for all the systems investigated here when considering the cold regimes typical of interstellar media.

The present formulation also permits a direct evaluation of the long-range dispersion coefficient $C_{6}$, which controls the capture effect by the long-range forces. Such coefficients can be defined through the following sum:

$$
C_{6}=\sum_{i=1}^{4} n_{i} C_{6 i}
$$

where any partial $C_{6 i}$ contribution is obtained as $C_{6 i}=\varepsilon \cdot r_{m}^{6}$ from the parameter values reported in Tables $1-4$, and $n_{i}$ represents the number of interaction pairs of the same type. The $\mathrm{C}_{6}$ values obtained for the complete series of $\mathrm{Ng}-\mathrm{C}_{3} \mathrm{H}_{6} \mathrm{O}$ complexes are reported in Table 9 and show an increase with the increase of the $\mathrm{Ng}$ polarizability. The asymptotic long-range attractions predicted by such dispersion coefficients can be compared with those estimated by Blanco et al. for Ne-, Ar, and Kr- propylene oxide [23-25] which also scale consistently with the $\mathrm{Ng}$ polarizability. In all cases, the present approach predicts an absolute value of the long-range attraction about 30\% stronger than in [25]. This is to be expected because their estimation is based on the $D_{0}$ value rather than on $D_{e}$, which is most effectively related to the long-range attraction. Furthermore, another factor which leads to 
an underestimation is the fact that $\mathrm{D}_{\mathrm{e}}$ arises from the balance of attraction-repulsion of the various interaction pairs, while the total long-range attraction depends on the sum of the different attraction pair contributions, which are included in the present determination.

Table 9. Long-range isotropic $C_{6}$ dispersion coefficients calculated on the ILJ potential.

\begin{tabular}{cc}
\hline System & $\boldsymbol{C}_{\mathbf{6}}\left(\mathbf{1 0 ^ { 4 }} \mathbf{m e V} \cdot \AA^{\mathbf{6}}\right)$ \\
\hline $\mathrm{He}-\mathrm{C}_{3} \mathrm{H}_{6} \mathrm{O}$ & 1.65 \\
\hline $\mathrm{Ne}-\mathrm{C}_{3} \mathrm{H}_{6} \mathrm{O}$ & 3.65 \\
\hline $\mathrm{Ar}-\mathrm{C}_{3} \mathrm{H}_{6} \mathrm{O}$ & 11.80 \\
\hline $\mathrm{Kr}-\mathrm{C}_{3} \mathrm{H}_{6} \mathrm{O}$ & 17.43 \\
\hline $\mathrm{Xe}_{-} \mathrm{C}_{3} \mathrm{H}_{6} \mathrm{O}$ & 25.78 \\
\hline $\mathrm{Rn}-\mathrm{C}_{3} \mathrm{H}_{6} \mathrm{O}$ & 34.35 \\
\hline
\end{tabular}

\section{Final Remarks and Conclusions}

Potential energy surfaces describing the anisotropic interaction of noble gases (Ar, $\mathrm{Kr}, \mathrm{Xe}$, and $\mathrm{Rn}$ ) with propylene oxide have been constructed. The van der Waals type forces, that govern the intermolecular interaction, have been formulated according to the ILJ function, where all parameter values are predicted by a phenomenological approach, that exploits those obtained for $\mathrm{He}$ and $\mathrm{Ne}-\mathrm{C}_{3} \mathrm{H}_{6} \mathrm{O}$, and scales them for the polarizability change along the $\mathrm{Ng}$ group. Indeed, in our previous work on the interaction between $\mathrm{Ng}$ (He and $\mathrm{Ne}$ )-propylene oxide, the parameters (here used as references) have been tested and fine-tuned, exploiting the comparison against ab initio calculations and against experimental scattering data [28].

The minima predicted by the present approach have been confirmed by high level ab initio calculations and show that the adopted potential parameters, reported in Tables 1-4, allow a proper representation of the PES. Contour maps at selected $R$ values are plotted in Figures 3-10, where the MEP is also shown.

The features of the obtained PESs stimulate important considerations:

(1) The adopted method represents the intermolecular potential by an analytic formulation that makes use of few parameters, all having a well-defined physical meaning. This physically grounded restriction, at variance with most interpolation procedures, ensures the reliability of the interaction in the whole space of the relative configurations;

(2) The present method allows a direct evaluation of the long-range dispersion coefficient $C_{6}$, that controls the capture character of the long-range forces;

(3) All systems present five minima very close in energy. Therefore, the collision dynamics is likely to be determined from their overall contribution, as well as from the possibility of adiabatic inter-conversion between them, hindered by the energy barriers that increase with the noble gas mass, and as the $R$ distance decreases;

(4) For each system, the location of the minimum energy changes as the intermolecular distance varies, that is the most stable configuration at long range, can substantially differ from that exhibited at intermediate and short range. An important consequence is that a system, formed by the trapping long-range anisotropic attractive forces, can be channeled during the relaxation of its internal degrees of freedom via fast non-adiabatic cooling, in configurations that can differ from that of the PES global minimum;

(5) The dissociation energy of $\mathrm{Ar}$ - propylene oxide and $\mathrm{Kr}$-propylene oxide, estimated by Blanco et al. [25], is consistent with the well depth and geometries of the minima determined in the present work. Moreover, considerations made at the previous point 4 can also applied to this point;

(6) The analytical formulation of the PES allows the calculation of physical, dynamical, and spectroscopic properties of the systems, which selectively depend on the weak anisotropic forces, effective both at long and intermediate range of separation distances; 
(7) The same method can be applied, after parameters scaling for the change in polarizability, to the description of simple diatomic molecule-propylene oxide systems, when the diatom $\left(\right.$ as $\mathrm{H}_{2}, \mathrm{~N}_{2}$ and $\mathrm{O}_{2}$ ), with rotational levels following a Boltzmann distribution at room and higher temperature, rotates sufficiently faster than propylene oxide. Therefore, under such conditions, the diatom, which during the collision behaves as a pseudo-atom, interacts with propylene oxide with intermolecular forces basically of van der Waals nature. In particular, $\mathrm{N}_{2}$ and $\mathrm{O}_{2}$ would behave like Ar, as they show a similar isotropic polarizability component, while the polarizability of $\mathrm{H}_{2}$ suggests for this molecule an intermediate behavior between $\mathrm{Ne}$ and Ar. However, the anisotropic character of the intermolecular forces emerges at low temperature, which is when only slowly rotating diatoms are interacting with the propylene oxide. In this case, the complete potential formulation must also include the anisotropic contribution of the electrostatic components, as those arising from permanent molecular electric multipole interactions. The method can also be extended to more complex systems of interest for their chiral properties, like propylene oxide dimers.

(8) These potential energy surfaces can be used to evaluate and investigate possible collision alignment processes, basic for chiral discrimination in gaseous streams and vortices occurring in no equilibrium atmosphere environments [40];

(9) Finally, it is also worth pointing out that this methodology can be profitably used to preliminarily address the search of ab-initio stationary points in multidimensional surfaces for weakly bound systems. This might be a difficult task, as they require a higher level of theory than bound systems, and may present many minima, particularly when some of them are shallow and can easily escape a grid search. The ab-initio calculation of transition states may be even more elusive. A preliminary detailed search on the present PES is reliable and much faster and allows identification of confined regions where minima or transition state points are located, strongly reducing the number of points to be evaluated quantum mechanically. Moreover, its formulation permits both forces and force constants to be obtained analytically, speeding up their calculation in molecular dynamics simulations, where these quantities play a crucial role, therefore alleviating the related computational burden.

Supplementary Materials: The following supporting information can be downloaded at: https:// www.mdpi.com/article/10.3390/sym14020249/s1. The Supplementary Information contains figures of the minimum configurations for $\mathrm{Kr}-, \mathrm{Xe}-$, and $\mathrm{Rn}$ - propylene oxide. Figure S1: Minima geometries for $\mathrm{Kr}$-propylene oxide identified on the present PES. The numbering of the $\mathrm{Kr}$ atom corresponds to the minima reported in Table 6. Figure S2: Minima geometries for Xe-propylene oxide identified on the present PES. The numbering of the Xe atom corresponds to the minima reported in Table 7. Figure S3: Minima geometries for Rn-propylene oxide identified on the present PES. The numbering of the $\mathrm{Rn}$ atom corresponds to the minima reported in Table 8. Figure S4: Color contour map for the Ar-propylene oxide system at $R=3.77 \AA$ (left panel). The axes report the angles $\theta$ and $\phi$ in degrees, while a color scale reports the potential energy in meV. Figure S5: Color contour map for the $\mathrm{Kr}$ -propylene oxide system at $R=3.88 \AA$ (left panel). The axes report the angles $\theta$ and $\phi$ in degrees, while a color scale reports the potential energy in meV. Table S1: Binding energies for Ar-propylene oxide system calculated at different levels of theory, MP2/CBS extrapolation and CCSD(T) correction. Table S2: Binding energies for Kr-propylene oxide system calculated at different levels of theory, MP2/CBS extrapolation and CCSD(T) correction. Table S3: Binding energies for Xe-propylene oxide system calculated at different levels of theory, MP2/CBS extrapolation and CCSD(T) correction. Table S4: Binding energies for Rn-propylene oxide system calculated at different levels of theory, $\mathrm{MP} 2 / \mathrm{CBS}$ extrapolation and CCSD(T) correction.

Author Contributions: All the authors have equally contributed to planning and writing the paper. All authors have read and agreed to the published version of the manuscript.

Funding: This research was funded by the Italian Ministry for Education, University and Research (MIUR) through SIR 2014, Scientific Independence of young Researchers (RBSI14U3VF).

Institutional Review Board Statement: Not applicable. 
Informed Consent Statement: Not applicable.

Data Availability Statement: The data that supports the findings of this study are available within the article and its supplementary material.

Acknowledgments: Cecilia Coletti and Alessandro Marrone gratefully acknowledge CINECA for granting computational time on Galileo100 through the ISCRA C program, code: HP10CT0CQ8.

Conflicts of Interest: The authors declare no conflict of interest.

\section{References}

1. Falcinelli, S.; Cappelletti, D. Special issue "Long-Range Intermolecular Interactions in chemistry and Physics". Chem. Phys. Lett. 2021, 786, 139190 .

2. Hong, Q.; Bartolomei, M.; Esposito, F.; Coletti, C.; Sun, Q.; Pirani, F. Reconciling experimental and theoretical vibrational deactivation in low energy $\mathrm{O}+\mathrm{N}_{2}$ collisions. Phys. Chem. Chem. Phys. 2021, 23, 15475-15479. [CrossRef] [PubMed]

3. Hong, Q.; Sun, Q.; Pirani, F.; Valentín-Rodríguez, M.A.; Hernández-Lamoneda, R.; Coletti, C.; Hernández, M.I.; Bartolomei, M. Energy exchange rate coefficients from vibrational inelastic $\mathrm{O}_{2}\left({ }^{3} \Sigma_{g}^{-}+\mathrm{O}_{2}\left({ }^{3} \Sigma_{g}^{-}\right.\right.$collisions on a new spin-averaged potential energy surface. J. Chem. Phys. 2021, 154, 064304. [CrossRef] [PubMed]

4. McGuire, B.A.; Carroll, P.B.; Loomis, R.A.; Finneran, I.A.; Jewell, P.R.; Remijan, A.J.; Blake, G.A. Discovery of the interstellar chiral molecule propylene oxide $\left(\mathrm{CH}_{3} \mathrm{CHCH}_{2} \mathrm{O}\right)$. Science 2016, 352, 1449-1452. [CrossRef]

5. Palazzetti, F.; Maciel, G.S.; Lombardi, A.; Grossi, G.; Aquilanti, V. The astrochemical observatory: Molecules in the laboratory and in the cosmos. J. Chin. Chem. Soc. 2013, 59, 1045-1052. [CrossRef]

6. Aquilanti, V.; Grossi, G.; Lombardi, A.; Maciel, G.S.; Palazzetti, F. The origin of chiral discrimination: Supersonic molecular beam experiments and molecular dynamics simulations of collisional mechanisms. Phys. Scr. 2008, 78, 058119. [CrossRef]

7. Quack, M. How important is parity violation for molecular and biomolecular chirality? Angew. Chem. Int. Ed. Engl. 2002, 41, 4618-4630. [CrossRef]

8. Lombardi, A.; Palazzetti, F.; Maciel, G.S.; Aquilanti, V.; Sevryuk, M.B. Simulation of oriented collision dynamics of simple chiral molecules. Int. J. Quant. Chem. 2011, 111, 1651-1658. [CrossRef]

9. Swalen, J.D.; Herschbach, D.R. Internal Barrier of Propylene Oxide from the Microwave Spectrum. I. J. Chem. Phys. 1957, 27, 100. [CrossRef]

10. Herschbach, D.R.; Swalen, J.D. Internal Barrier of Propylene Oxide from the Microwave Spectrum. II. J. Chem. Phys. 1958, 29, 761. [CrossRef]

11. Kawiecki, R.W.; Devlin, F.; Stephens, P.J.; Amos, R.D.; Handy, N.C. Vibrational circular dichroism of propylene oxide. Chem. Phys. Lett. 1988, 145, 411-417. [CrossRef]

12. Šebestik, J.; Bouř, P. Raman Optical Activity of Methyloxirane Gas and Liquid. J. Phys. Chem. Lett. 2011, 2, 498-502. [CrossRef]

13. Merten, C.; Bloino, J.; Barone, V.; Xu, Y. Anharmonicity effects in the vibrational CD spectra of propylene oxide. J. Phys. Chem. Lett. 2013, 4, 3424-3428. [CrossRef]

14. Che, D.-C.; Palazzetti, F.; Okuno, Y.; Aquilanti, V.; Kasai, T. Electrostatic hexapole state-selection of the asymmetric-top molecule propylene oxide. J. Phys. Chem. A 2010, 114, 3280. [CrossRef] [PubMed]

15. Che, D.-C.; Kanda, K.; Palazzetti, F.; Aquilanti, V.; Kasai, T. Electrostatic Hexapole State-Selection of the Asymmetric-Top Molecule Propylene Oxide: Rotational and Orientational Distributions. Chem. Phys. 2012, 399, 180-192. [CrossRef]

16. Falcinelli, S.; Vecchiocattivi, F.; Alagia, M.; Schio, L.; Richter, R.; Stranges, S.; Catone, D.; Arruda, M.S.; Mendes, L.A.V.; Palazzetti, F.; et al. Double photoionization of propylene oxide: A coincidence study of the ejection of a pair of valence-shell electrons. J. Chem. Phys. 2018, 148, 114302. [CrossRef]

17. Falcinelli, S.; Rosi, M.; Pirani, F.; Bassi, D.; Alagia, M.; Schio, L.; Richter, R.; Stranges, S.; Balucani, N.; Lorent, V.; et al. Angular distribution of ion products in the double photoionization of propylene oxide. Front. Chem. 2019, 7, 621. [CrossRef]

18. Dubnikova, F.; Lifshitz, A. Isomerization of Propylene Oxide. Quantum Chemical Calculations and Kinetic Modeling. J. Phys. Chem. A 2000, 104, 4489-4496. [CrossRef]

19. Elango, M.; Maciel, G.S.; Palazzetti, F.; Lombardi, A.; Aquilanti, V. Quantum Chemistry of $\mathrm{C}_{3} \mathrm{H}_{6} \mathrm{O}$ Molecules: Structure and Stability, Isomerization Pathways, and Chirality Changing Mechanisms. J. Phys. Chem. A 2010, 114, 9864-9874. [CrossRef]

20. Witt, A.N. The Chemical Composition of the Interstellar Medium. Phil. Trans. Math Phys. Eng. Sci. 2001, 359, 1949-1959. [CrossRef]

21. McGuire, B.A. 2018 Census of Interstellar, Circumstellar, Extragalactic, Protoplanetary Disk, and Exoplanetary Molecules. Astrophys. J. 2018, 239, 17. [CrossRef]

22. Orek, C.; Kłos, J.; Lique, F.; Bulut, N. Ab initio studies of the $\operatorname{Rg}_{-} \mathrm{NO}^{+}\left(\mathrm{X}^{1} \Sigma^{+}\right)$van der Waals complexes $(\mathrm{Rg}=\mathrm{He}, \mathrm{Ne}, \mathrm{Ar}, \mathrm{Kr}, \mathrm{and}$ Xe). J. Chem. Phys. 2016, 144, 204303. [CrossRef] [PubMed]

23. Blanco, S.; Maris, A.; Melandri, S.; Caminati, W. Rotational spectrum of propylene oxide-neon. Mol. Phys. 2002, 100, 3245-3249. [CrossRef]

24. Blanco, S.; Maris, A.; Millemaggi, A.; Caminati, W. The most stable conformer of the propylene oxide-argon complex. J. Mol. Struct. 2002, 612, 309-313. [CrossRef] 
25. Blanco, S.; Melandri, S.; Maris, A.; Caminati, W.; Velino, B.; Kisiel, Z. Free jet rotational spectrum of propylene oxide-krypton and modelling and ab initio calculations for propylene oxide-rare gas dimers. Phys. Chem. Chem. Phys. 2003, 5, 1359-1364. [CrossRef]

26. Su, Z.; Borho, N.; Xu, Y. Chiral Self-Recognition: Direct Spectroscopic Detection of the Homochiral and Heterochiral Dimers of Propylene Oxide in the Gas Phase. J. Am. Chem. Soc. 2006, 128, 17126-17131. [CrossRef]

27. Faure, A.; Dagdigian, P.J.; Rist, C.; Dawes, C.; Qintas-Sànchez, E.; Lique, F.; Hochlaf, M. Interaction of Chiral Propylene Oxide $\left(\mathrm{CH}_{3} \mathrm{CHCH}_{2} \mathrm{O}\right)$ with Helium: Potential Energy Surface and Scattering Calculations. ACS Earth Space Chem. 2019, 3, 964-972. [CrossRef]

28. Palazzetti, F.; Cappelletti, D.; Coletti, C.; Falcinelli, S.; Pirani, F. Molecular Beam Scattering Experiments on Noble Gas-Propylene Oxide: Total Integral Cross Sections and Potential Energy Surfaces of He-and $\mathrm{Ne}_{-} \mathrm{C}_{3} \mathrm{H}_{6}$ O. J. Chem. Phys. 2021, $155,234301$. [CrossRef]

29. Lombardi, A.; Palazzetti, F. Chirality in molecular collision dynamics. J. Phys. Cond. Matt. 2018, 30, 063003. [CrossRef]

30. Kiss, B.; Szőri, M.; Jedlovszky, P. Adsorption of Propylene Oxide on Amorphous Ice under Interstellar Conditions. A Grand Canonical Monte Carlo Simulation Study. J. Phys. Chem. C 2020, 124, 16402-16414. [CrossRef]

31. Capitelli, M.; Cappelletti, D.; Colonna, G.; Gorse, C.; Laricchiuta, A.; Liuti, G.; Longo, S.; Pirani, F. On the possibility of using model potentials for collision integral calculations of interest for planetary atmospheres. Chem. Phys. 2007, 338, 62-68. [CrossRef]

32. Cambi, R.; Cappelletti, D.; Pirani, F.; Liuti, G. Generalized correlations in terms of polarizability for van der Waals interaction potential parameter calculations. J. Chem. Phys. 1991, 95, 1852-1861. [CrossRef]

33. Pirani, F.; Cappelletti, D.; Liuti, G. Range strength and anisotropy of intermolecular forces in atom-molecule systems: An atom-bond pairwise additivity approach. Chem. Phys. Lett. 2001, 350, 286-296. [CrossRef]

34. Pirani, F.; Maciel, G.S.; Cappelletti, D.; Aquilanti, V. Experimental benchmarks and phenomenology of interatomic forces: Open shell and electronic anisotropy effects. Int. Rev. Phys. Chem. 2006, 25, 165-199. [CrossRef]

35. Olney, T.N.; Cann, C.G.; Cooper, G.; Brion, C.E. Absolute scale determination for photoabsorpion spectra and the calculation of molecular properties using dipole sum-rules. Chem. Phys. 1997, 223, 59-98. [CrossRef]

36. Frisch, M.J.; Trucks, G.W.; Schlegel, H.B.; Scuseria, G.E.; Robb, M.A.; Cheeseman, J.R.; Scalmani, G.; Barone, V.; Petersson, G.A.; Nakatsuji, H.; et al. (Eds.) Gaussian 16, Revision C.01; Gaussian, Inc.: Wallingford, CT, USA, 2016.

37. Peterson, K.A.; Woon, D.E.; Dunning, T.H., Jr. Benchmark calculations with correlated molecular wave functions. IV. The classical barrier height of the $\mathrm{H}+\mathrm{H} 2 \rightarrow \mathrm{H} 2+\mathrm{H}$ reaction. J. Chem. Phys. 1994, 100, 7410. [CrossRef]

38. Boys, S.F.; Bernardi, R. The calculation of small molecular interactions by the differences of separate total energies. Some procedures with reduced errors. Mol. Phys. 1970, 19, 553. [CrossRef]

39. Coletti, C.; Re, N. High Level Theoretical Study of Benzene-Halide Adducts: The Importance of C-H-Anion Hydrogen Bonding. J. Phys. Chem. A 2009, 113, 1578-1585. [CrossRef]

40. Lee, H.-N.; Chang, L.-C.; Su, T.-M. Optical rotamers of substituted simple alkanes induced by macroscopic translation-rotational motions. Chem. Phys. Lett. 2011, 507, 63-68. [CrossRef] 